\title{
Bladder Neuroendocrine Neoplasm
}

National Cancer Institute

\section{Source}

National Cancer Institute. Bladder Neuroendocrine Neoplasm. NCI Thesaurus. Code C157758.

A neuroendocrine neoplasm that arises from the bladder. 